\title{
Coefficient inequalities for certain subclasses of multivalent functions associated with conic domain
}

\author{
Muhammad Sabil Ur Rehman', Qazi Zahoor Ahmad ${ }^{1,2^{*}}$, H.M. Srivastava ${ }^{3,4,5}$, Nazar Khan', \\ Maslina Darus ${ }^{6}$ and Muhammad Tahir ${ }^{1}$
}

"Correspondence:

zahoorqazi5@gmail.com

1 Department of Mathematics,

Abbottabad University of Science and Technology, Abbottabad

22010, Pakistan

${ }^{2}$ Government Akhtar Nawaz Khan

(Shaheed) Degree College, KTS,

Haripur 22620, Pakistan

Full list of author information is

available at the end of the article

\begin{abstract}
A number of families of $q$-extensions of analytic functions in the open unit disk $\mathbb{U}$ have been defined by means of basic (or $q$-)calculus and considered from many distinctive prospectives and viewpoints. In this paper, we generalize and study certain subclasses of analytic functions involving higher-order $q$-derivative operators. We settle characteristic equations for these presumably new classes and also study numerous coefficient inequalities. For the results obtained in this presentation, we also carry out appropriate connections with those in multiple other concerning works on this subject.
\end{abstract}

MSC: Primary 05A30; 30C45; secondary 11B65; 47B38

Keywords: Analytic functions; Univalent functions; Convex and $q$-convex functions; Starlike and $q$-starlike functions; $q$-Derivative operator; $q$-Hypergeometric functions

\section{Introduction and definitions}

By $\mathcal{A}(p)$ we denote the class of functions with series representation

$$
f(z)=z^{p}+\sum_{n=1}^{\infty} a_{n+p} z^{n+p} \quad(p \in \mathbb{N}=\{1,2, \ldots\})
$$

which is analytic and $p$-valent in the open unit disk

$$
\mathbb{U}=\{z: z \in \mathbb{C} \text { and }|z|<1\} .
$$

In particular, we denote

$$
\mathcal{A}:=\mathcal{A}(1)
$$

Moreover, we denote by $\mathcal{S} \subset \mathcal{A}$ the class of all univalent functions in the unit disk $\mathbb{U}$.

c) The Author(s) 2020. This article is licensed under a Creative Commons Attribution 4.0 International License, which permits use, sharing, adaptation, distribution and reproduction in any medium or format, as long as you give appropriate credit to the original author(s) and the source, provide a link to the Creative Commons licence, and indicate if changes were made. The images or other third party material in this article are included in the article's Creative Commons licence, unless indicated otherwise in a credit line to the material. If material is not included in the article's Creative Commons licence and your intended use is not permitted by statutory regulation or exceeds the permitted use, you will need to obtain permission directly from the copyright holder. To view a copy of this licence, visit http://creativecommons.org/licenses/by/4.0/. 
For two analytic functions $f_{j}, j=1,2$, in $\mathbb{U}$, a function $f_{1}$ is said to be subordinate to the function $f_{2}$ and write as

$$
f_{1} \prec f_{2} \quad \text { or } \quad f_{1}(z) \prec f_{2}(z)
$$

if in $\mathbb{U}$, we can find an analytic Schwarz function $w$ with

$$
w(0)=0 \quad \text { and } \quad|w(z)|<1
$$

such that

$$
f_{1}(z)=f_{2}(w(z)) .
$$

Further, if the function $f_{2}$ is univalent in $\mathbb{U}$ then following equivalence relation holds true

$$
f_{1}(z) \prec f_{2}(z) \quad(z \in \mathbb{U}) \quad \Rightarrow \quad f_{1}(0)=f_{2}(0) \quad \text { and } \quad f_{1}(\mathbb{U}) \subset f_{2}(\mathbb{U})
$$

The noteworthy class of Carathéodory functions $\mathcal{P}$ consists of all analytic functions $\psi$ in $\mathbb{U}$ normalized by

$$
\psi(z)=1+\sum_{n=1}^{\infty} \psi_{n} z^{n}
$$

and satisfying

$$
\Re(\psi(z))>0 \quad(\forall z \in \mathbb{U}) .
$$

For a convex function $f$, it is always true that the image of $f$ under $\mathbb{U}$ and all circles lying within $\mathbb{U}$ centered at the origin are convex arcs. But justification is required whether the characteristic still holds for circles with center at any other point, say $\xi$. Goodman $[4,5]$ answered negatively and defined uniformly convex and starlike functions that have this nice characteristic. Analytically, he defined uniformly convex and starlike functions, respectively, as

$$
\Re\left\{1+\frac{(z-\xi) f^{\prime \prime}(z)}{f^{\prime}(z)}\right\}>0 \quad(z \in \mathbb{U})
$$

and

$$
\Re\left\{1+\frac{(z-\xi) f^{\prime}(z)}{f^{\prime}(z)-f^{(\xi)}}\right\}>0 \quad(z \in \mathbb{U}) .
$$

We denote the former by $\mathcal{U C} \mathcal{V}$ and the later by $\mathcal{U S} * \mathcal{T}$. It is natural to ask whether classical Alexander's result holds for these two classes, but there are counterexamples [4], which show that the relation is not true for these classes. Rønning [24], using $\mathcal{U C V}$, introduced the class

$$
\mathcal{S}^{*} \mathcal{T}=\left\{f \in \mathcal{A}: f(z)=z g^{\prime}(z), g(z) \in \mathcal{U C V}\right\}
$$


and succeeded in proving that neither $\mathcal{S}^{*} \mathcal{T} \not \subset \mathcal{U} \mathcal{S}^{*} \mathcal{T}$ nor $\mathcal{U S} \mathcal{S}^{*} \mathcal{T} \not \subset \mathcal{S}^{*} \mathcal{T}$. Ultimately, Rønning [23] and Ma and Minda [14] introduced the following one-variable characterization of these classes.

Definition 1 Let $f \in \mathcal{A}$. Then $f \in \mathcal{U C V}$ if

$$
\Re\left\{\frac{\left(z f^{\prime}(z)\right)^{\prime}}{f^{\prime}(z)}\right\}>\left|\frac{z f^{\prime \prime}(z)}{f^{\prime}(z)}\right| \quad(z \in \mathbb{U})
$$

Definition 2 Let $f \in \mathcal{A}$. Then $f \in \mathcal{S}^{*} \mathcal{T}$ if

$$
\Re\left\{\frac{z f^{\prime}(z)}{f(z)}\right\}>\left|\frac{z f^{\prime}(z)}{f(z)}-1\right| \quad(z \in \mathbb{U})
$$

This led to the basis for conic domains introduced by Kanas and Wiśniowska [10,11] as

$$
\Omega_{k}=\left\{u+i v: u>k \sqrt{(u-1)^{2}+v^{2}}\right\}, \quad k \geqq 0 .
$$

These domains represent the right half-plane, a parabola, a hyperbola, and an ellipse for $k=0, k=1,0<k<1$, and $k>1$, respectively.

The role of an extremal function is done by the function $p_{k}(z)$ given by

$$
p_{k}(z)= \begin{cases}\frac{1+z}{1-z} & (k=0), \\ 1+\frac{2}{\pi^{2}}\left(\log \frac{1+\sqrt{z}}{1-\sqrt{z}}\right)^{2} & (k=1), \\ 1+\frac{2}{1-k^{2}} \sinh ^{2}\left\{\left(\frac{2}{\pi} \arccos k\right) \arctan h \sqrt{z}\right\} & (0 \leq k<1), \\ 1+\frac{1}{k^{2}-1}\left[1+\sin \left(\frac{\pi}{2 K(\kappa)} \int_{0}^{\frac{u(z)}{\sqrt{k}}} \frac{d t}{\sqrt{1-t^{2}} \sqrt{1-\kappa^{2} t^{2}}}\right)\right] & (k>1),\end{cases}
$$

where

$$
u(z)=\frac{\sqrt{\kappa}-z}{\sqrt{\kappa} z-1} \quad(\forall z \in \mathbb{U})
$$

and $\kappa \in(0,1)$ is chosen so that

$$
k=\cosh \left(\pi K^{\prime}(\kappa) /(4 K(\kappa))\right),
$$

where $K(\kappa)$ denotes the first-kind Legendre complete elliptic integral, and its derivative is given by

$$
K^{\prime}(\kappa)=K\left(\sqrt{1-\kappa^{2}}\right)
$$

and called the complementary integral of $K(t)$. If we set the function

$$
p_{k}(z)=1+\delta_{k} z+\cdots,
$$


subsequently, by [9] it is obvious that from (1.4) we have

$$
\delta_{k}= \begin{cases}\frac{8(\arccos k)^{2}}{\pi\left(1-k^{2}\right)} & (0 \leq k<1), \\ \frac{8}{\pi^{2}} & (k=1), \\ \frac{\pi^{2}}{4 k^{2}(1+t) \sqrt{t} R^{2}(t)} & (k>1) .\end{cases}
$$

Subjected to the above-mentioned conic domain, we define some elementary classes.

Definition 3 Let $f$ be a function from the functional class $\mathcal{A}$. Then $f \in k-\mathcal{U C V}$ if

$$
\Re\left\{\frac{\left(z f^{\prime}(z)\right)^{\prime}}{f^{\prime}(z)}\right\}>k\left|\frac{z f^{\prime \prime}(z)}{f^{\prime}(z)}\right| \quad(\forall z \in \mathbb{U} \text { and } k \geq 0) .
$$

Definition 4 A normalized analytic function $f$ belongs to the class $k-\mathcal{S}^{*} \mathcal{T}$ if

$$
\Re\left\{\frac{z f^{\prime}(z)}{f(z)}\right\}>k\left|\frac{z f^{\prime}(z)}{f(z)}-1\right| \quad(\forall z \in \mathbb{U} \text { and } k \geq 0) .
$$

Definition 5 Let $f$ be a function from the functional class $\mathcal{A}$. Then $f \in k-\mathcal{U Q}$ if there exists a function $g \in k-\mathcal{U C V}$ such that

$$
\Re\left\{\frac{\left(z f^{\prime}(z)\right)^{\prime}}{g^{\prime}(z)}\right\}>k\left|\frac{z f^{\prime \prime}(z)}{g^{\prime}(z)}\right| \quad(\forall z \in \mathbb{U} \text { and } k \geq 0) .
$$

Definition 6 A normalize analytic function $f$ belongs to the class $k-\mathcal{U} \mathcal{K}$ if there exists a function $g \in k-\mathcal{S}^{*} \mathcal{T}$ such that

$$
\Re\left\{\frac{z f^{\prime}(z)}{g(z)}\right\}>k\left|\frac{z f^{\prime}(z)}{g(z)}-1\right| \quad(\forall z \in \mathbb{U} \text { and } k \geq 0) .
$$

Now we recall some firm footing concept details and definitions of the $q$-difference calculus, which play a vital role in our presentation. Unless otherwise notified, we presume that $0<q<1$ and $p \in \mathbb{N}=\{1,2,3, \ldots\}$. For a nonnegative number $\lambda$, the $q$-number $[\lambda]_{q}$ is defined by

$$
[\lambda]_{q}=\sum_{j=0}^{j-1} q^{j}=1+q+q^{2}+\cdots+q^{j-1}, \quad[0]_{q}=0
$$

In general, for $\lambda \in \mathbb{C}$, we have $[\lambda]_{q}=\frac{1-q^{\lambda}}{1-q}$. The $q$-factorials $[j]_{q}$ ! are defined by $[0]_{q} !=0$ and $[j]_{q} !=\prod_{k=1}^{j}[k]_{q}$. It is straightforward to observe that $\lim _{q \rightarrow 1-}[\lambda]_{q}=\lambda$ and $\lim _{q \rightarrow 1-}[j]_{q} !=j !$.

Definition 7 (See [7] and [8]) For a function $f$ from class $\mathcal{A}$, the $q$-derivative (or $q$ difference) operator $D_{q}$ in a subset of complex numbers $\mathbb{C}$ is defined by

$$
\left(D_{q} f\right)(z)= \begin{cases}\frac{f(z)-f(q z)}{(1-q) z} & (z \neq 0) \\ f^{\prime}(0) & (z=0)\end{cases}
$$

provided that $f^{\prime}(0)$ exists. 
We observe from Definition 7 that

$$
\lim _{q \rightarrow 1-}\left(D_{q} f\right)(z)=\lim _{q \rightarrow 1-} \frac{f(z)-f(q z)}{(1-q) z}=f^{\prime}(z)
$$

for a differentiable function $f$ in a subset of $\mathbb{C}$. Further, by (1.1) and (1.6) we obtain

$$
\begin{aligned}
& \left(D_{q}^{(1)} f\right)(z)=[p]_{q} z^{p-1}+\sum_{n=1}^{\infty}[n+p]_{q} a_{n+p} z^{n+p-1} \\
& \left(D_{q}^{(2)} f\right)(z)=[p]_{q}[p-1]_{q} z^{p-2}+\sum_{n=1}^{\infty}[n+p]_{q}[n+p-1]_{q} a_{n+p} z^{n+p-2}, \\
& \left(D_{q}^{(p)} f\right)(z)=[p]_{q} !+\sum_{n=1}^{\infty} \frac{[n+p]_{q} !}{[n]_{q} !} a_{n+p} z^{n},
\end{aligned}
$$

where $\left(D_{q}^{(p)} f\right)(z)$ is the $p$ th $q$-derivative of $f(z)$.

Recently, the studies of $q$-calculus have inspired an intense interest of researchers because of its advantages in many areas of mathematics and physics. The significance of the operator $D_{q}$ is quite obvious by its applications in the study of several subclasses of analytic functions. Initially, in 1990, Ismail et al. [6] gave the idea of $q$-extension of the class of starlike functions; nevertheless, a foothold usage of the $q$-calculus in the context of geometric function theory was effectively invoked by Srivastava [26]. After that, wonderful studies have been done by numerous mathematicians offering a momentous part in the advancement of geometric function theory. In particular, the study the $q$-Mittag-Leffler functions for close-to-convex functions was done by Srivastava and Bansal [30] (see also [21]). In [32], they also considered the functional class of $q$-starlike functions related to conic region $\sigma_{k}$, where the estimate of the third Hankel determinant has been settled in [17] (see also [28]). Recently, Srivastava et al. (see, e.g., [15, 31, 34, 35] published a set of papers, in which they concentrated on the class of $q$-starlike functions related to the Janowski functions from different aspects. For some more recent investigations on $q$-calculus, we refer to $[13,16,27,29,33]$. In this paper, we mainly generalize the work presented in Srivastava et al. [32].

Definition 8 (See [6]) A function $f \in \mathcal{A}$ belongs to the functional class $\mathcal{S}_{q}^{*}$ if

$$
f(0)=f^{\prime}(0)-1=0
$$

and

$$
\left|\frac{z}{f(z)}\left(D_{q} f\right) z-\frac{1}{1-q}\right| \leq \frac{1}{1-q} .
$$

In view of the last inequality, it is obvious that, in the limiting case $q \rightarrow 1-$,

$$
\left|w-\frac{1}{1-q}\right| \leq \frac{1}{1-q},
$$


the above closed disk is merely the right-half plane, and the class $\mathcal{S}_{q}^{*}$ of $q$-starlike functions turns into the prominent class $\mathcal{S}^{*}$. Analogously, by the principle of subordination we may express relations (1.10) and (1.11) as follows (see [36]):

$$
\frac{z}{f(z)}\left(D_{q} f\right)(z) \prec \widehat{p}(z) \quad\left(\widehat{p}(z)=\frac{1+z}{1-q z}\right) .
$$

The notation $\mathcal{S}_{q}^{*}$ was first used by Sahoo et al. [25].

Remark 1 In defining the class $\mathcal{C}_{q}$ of $q$-convex functions, the most important Alexander theorem [3] for functions $f \in \mathcal{A}$ was used by Baricz and Swaminathan [2] as

$$
f(z) \in \mathcal{C}_{q} \quad \Longleftrightarrow \quad z\left(D_{q} f\right)(z) \in \mathcal{S}_{q}^{*}
$$

The generalization of $\mathcal{P}\left(p_{k}\right)$ to $k-\mathcal{P}_{q}$ presented in Definition 9 is due to Srivastava et al. [32]. He used the conic domain and the earlier discussed $q$-calculus as follows.

Definition 9 A function $\psi \in \mathcal{P}$ belongs to the class $k-\mathcal{P}_{q}$ if the following relation holds:

$$
\psi(z) \prec \frac{2 p_{k}(z)}{(1+q)+(1-q) p_{k}(z)}
$$

with the function $p_{k}(z)$ given in equation (1.4).

It is interesting that in geometric characteristics the function $\psi \in k-\mathcal{P}_{q}$ takes all values from the domain $\Omega_{k, q}, k \geq 0$, analytically given by

$$
\Omega_{k, q}=\left\{w: \Re\left(\frac{(1+q) w}{(q-1) w+2}\right)>k\left|\frac{(1+q) w}{(q-1) w+2}-1\right|\right\}
$$

which represent $q$-analogues of generalized conic regions.

Note the following easily observable facts about the class $k-\mathcal{P}_{q}$.

Remark 2 Firstly, we see that

$$
k-\mathcal{P}_{q} \subseteq \mathcal{P}\left[\frac{2 k}{2 k+1+q}\right]
$$

where $\mathcal{P}\left[\frac{2 k}{2 k+1+q}\right]$ is the famous class of functions with real parts greater than $\frac{2 k}{2 k+1+q}$. Secondly, we have

$$
\lim _{q \rightarrow 1-} k-\mathcal{P}_{q}=\mathcal{P}\left(p_{k}\right)
$$

where $\mathcal{P}\left(p_{k}\right)$ is the familiar class given by Wisniowska and Kanas [10]. Thirdly, by taking the limit we obtain

$$
\lim _{q \rightarrow 1-} 0-\mathcal{P}_{q}=\mathcal{P},
$$

where $\mathcal{P}$ is the class of functions given by (1.2). 
Using the $q$-differential operator, various new classes have been defined. Hence it is natural to give the following definition.

Definition 10 A function $f \in \mathcal{A}$ is said to belong to the class $k-\mathcal{S}_{q}^{*}(p)$ if

$$
\Re\left(\frac{(1+q) \frac{z\left(D_{q}^{(p)} f\right)(z)}{\left(D_{q}^{(p-1)} f\right)(z)}}{(q-1) \frac{z\left(D_{q}^{(p)} f\right)(z)}{\left(D_{q}^{(p-1)} f\right)(z)}+2}\right)>k\left|\frac{(1+q) \frac{z\left(D_{q}^{(p)} f\right)(z)}{\left(D_{q}^{(p-1)} f\right)(z)}}{(q-1) \frac{z\left(D_{q}^{(p)} f\right)(z)}{\left(D_{q}^{(p-1)} f\right)(z)}+2}-1\right|
$$

or, equivalently,

$$
\frac{z\left(D_{q}^{(p)} f\right)(z)}{\left(D_{q}^{(p-1)} f\right)(z)} \in k-\mathcal{P}_{q}
$$

Remark 3 First of all, it is straightforward to see that

$$
0-\mathcal{S}_{q}^{*}(1)=\mathcal{S}_{q}^{*}
$$

where the functional class $\mathcal{S}_{q}^{*}$ was considered and analyzed by Ismail et al. [6]. Secondly, we easily observe that

$$
\lim _{q \rightarrow 1-} k-\mathcal{S}_{q}^{*}(1)=k-\mathcal{S}^{*}=k-\mathcal{S}^{*} \mathcal{T}
$$

where the class $k-\mathcal{S}^{*} \mathcal{T}$ was presented and studied by Kanas and Wiśniowska [11]. Thirdly,

$$
k-\mathcal{S}_{q}^{*}(1)=k-\mathcal{S}_{q}^{*}
$$

where the function class $k-\mathcal{S}_{q}^{*}$ was initially considered and studied by Srivastava et al. [32]. Finally,

$$
\lim _{q \rightarrow 1-} 0-\mathcal{S}_{q}^{*}(1)=\mathcal{S}^{*}
$$

where $\mathcal{S}^{*}$ is the essential class of starlike functions.

Definition 11 Just as in Remark 1, by the Alexander theorem [3] the class $k-\mathcal{C}_{q}$ can be defined by the following relation:

$$
f(z) \in k-\mathcal{C}_{q}(p) \quad \Longleftrightarrow \quad \frac{z}{p !}\left(D_{q}^{(p)} f\right)(z) \in k-\mathcal{S}_{q}^{*}(p) .
$$

Definition 12 Any function $f \in \mathcal{A}(p)$ is said to belong to the class $k-\mathcal{K}_{q}(p)$ if

$$
\Re\left(\frac{(1+q) \frac{z\left(D_{q}^{(p)} f\right)(z)}{\left(D_{q}^{(p-1)} g\right)(z)}}{(q-1) \frac{z\left(D_{q}^{(p)} f\right)(z)}{\left(D_{q}^{(p-1)} g\right)(z)}+2}\right)>k\left|\frac{(1+q) \frac{z\left(D_{q}^{(p)} f\right)(z)}{\left(D_{q}^{(p-1)} g\right)(z)}}{(q-1) \frac{z\left(D_{q}^{(p)} f\right)(z)}{\left(D_{q}^{(p-1)} g\right)(z)}+2}-1\right|
$$


or, equivalently,

$$
\frac{z\left(D_{q}^{(p)} f\right)(z)}{\left(D_{q}^{(p-1)} g\right)(z)} \in k-\mathcal{P}_{q}
$$

for some $g \in k-\mathcal{S}_{q}^{*}(p)$.

Definition 13 In similar manner as in Remark 1, by using the idea of Alexander's relation [3] we define the class $k-\mathcal{C}_{q}^{*}(p)$ by the following relation:

$$
f(z) \in k-\mathcal{C}_{q}^{*}(p) \quad \Longleftrightarrow \frac{z}{p !}\left(D_{q}^{(p)} f\right)(z) \in k-\mathcal{K}_{q}(p) .
$$

Remark 4 First of all, we can see that

$$
0-\mathcal{K}_{q}(1)=\mathcal{K}_{q},
$$

where $\mathcal{K}_{q}(p)$ is the function class defined and examined by Raghavendar et al. [20]. Secondly, in the limit case, we have

$$
\lim _{q \rightarrow 1-} k-\mathcal{K}_{q}(1)=k-\mathcal{K}=k-\mathcal{U} \mathcal{K} \quad \text { and } \quad \lim _{q \rightarrow 1-} k-\mathcal{C}_{q}^{*}(1)=k-\mathcal{C}^{*}=k-\mathcal{U} \mathcal{Q}
$$

where $k-\mathcal{U} K(p)$ and $k-\mathcal{U} \mathcal{Q}(p)$ are the function classes introduced and studied by Acu [1]. Thirdly, we have

$$
\lim _{q \rightarrow 1-} 0-\mathcal{K}_{q}(1)=\mathcal{K} \quad \text { and } \quad \lim _{q \rightarrow 1-} 0-\mathcal{C}_{q}^{*}(1)=\mathcal{C}^{*}
$$

where $\mathcal{C}^{*}$ and $\mathcal{K}$ are the function classes of quasi-convex and close-to-convex functions; for details, see $[12,19]$.

\section{A set of lemmas}

Each of the lemmas given further will be helpful in demonstrating our main results.

Lemma 1 ([22]) Let $\psi$ be a function of the form

$$
\psi(z)=1+\sum_{n=1}^{\infty} \psi_{n} z^{n}
$$

subordinate to a function $\mathfrak{H}$ of the form

$$
\mathfrak{H}(z)=1+\sum_{n=1}^{\infty} C_{n} z^{n}
$$

In particular, when $\mathfrak{H}$ is univalent in the unit disk $\mathbb{U}$ and $\mathcal{H}(\mathbb{U})$ is convex, then

$$
\left|\psi_{n}\right| \leq\left|C_{1}\right| \quad(n \in \mathbb{N})
$$


Lemma 2 Suppose that the sequence $\left\{a_{k}\right\}_{k=0}^{\infty}$ is defined by

$$
a_{p}=1
$$

and

$$
a_{n+p}=\frac{\delta_{k}(q+1)[n+1]_{q} !}{2[n+p]_{q} !\left([n+1]_{q}-1\right)} \sum_{l=1}^{n-1} \frac{[p+l]_{q} !}{[l+1]_{q} !} a_{p+l} .
$$

Then

$$
a_{n+p-1}=\prod_{j=2}^{n} \frac{[j]_{q}\left\{2\left([j]_{q}-1\right)+\delta_{k}(q+1)\right\}}{2\left\{[j]_{q}-1\right\}[j+p-1]_{q}} .
$$

Proof By (2.1) we easily get

$$
\frac{[n+p]_{q} !}{[n+1]_{q} !}\left([n+1]_{q}-1\right) a_{n+p}=\sum_{l=1}^{n} \frac{[n+p-l]_{q} !}{[n+1-l]_{q} !} a_{n+p-l} c_{l}
$$

and

$$
\frac{[n+p-1]_{q} !}{[n+1]_{q} !}\left([n+1]_{q}-1\right) a_{n+p-1}=\sum_{l=1}^{n} \frac{[n+p-1-l]_{q} !}{[n+1-l]_{q} !} a_{n+p-1-l} c_{l} .
$$

Combining (2.2) and (2.3), we obtain

$$
\frac{a_{n+p}}{a_{n+p-1}}=\frac{[n+1]_{q}\left\{2\left([n]_{q}-1\right)+\delta_{k}(1+q)\right\}}{2\left\{\left\{[n+1]_{q}-1\right\}[n+p]_{q}\right.} .
$$

Similarly, we deduce the following result:

$$
a_{n+p-1}=\frac{a_{n+p-1}}{a_{n+p-2}} \cdot \frac{a_{n+p-2}}{a_{n+p-3}} \cdot \ldots \cdot \frac{a_{p+2}}{a_{p+1}} \cdot \frac{a_{p+1}}{a_{p}} \cdot a_{p} .
$$

This completes the proof of Lemma 2.

\section{Main results}

In this section, we prove our main results. We assume that

$$
k \geq 0 \text { and } q \in(0,1) .
$$

Theorem 1 Let $f$ be a p-valently analytic function of the form (1.1). Then $f$ belongs to the class $k-\mathcal{S}_{q}^{*}$ if it satisfies the condition

$$
\sum_{n=1}^{\infty} \frac{[n+p]_{q} !}{[n+1]_{q} !} \Lambda_{3}\left|a_{n+p}\right|<(1+q),
$$

where

$$
\Lambda_{3}=2(k+1) q\left([n+1]_{q}-1\right)+\left|(q-1)[n+1]_{q}+2\right| \text {. }
$$


Proof If (3.1) holds, then it suffices to establish the inequality

$$
k\left|\frac{(1+q) \frac{z\left(D_{q}^{(p)} f\right)(z)}{D_{q}^{(p-1)} f(z)}}{(q-1) \frac{z\left(D_{q}^{(p)} f\right)(z)}{D_{q}^{(p-1)} f(z)}+2}-1\right|-\Re\left[\frac{(1+q) \frac{z\left(D_{q}^{(p)} f\right)(z)}{D_{q}^{(p-1)} f(z)}}{(q-1) \frac{z\left(D_{q}^{(p)} f\right)(z)}{D_{q}^{(p-1)} f(z)}+2}-1\right]<1 .
$$

Since

$$
\begin{aligned}
k \mid & \frac{(1+q) \frac{z\left(D_{q}^{(p)} f\right)(z)}{\left(D_{q}^{(p-1)} f\right)(z)}}{(q-1) \frac{z\left(D_{q}^{(p)} f\right)(z)}{\left(D_{q}^{(p-1)} f\right)(z)}+2}-1 \mid-\Re\left[\frac{(1+q) \frac{z\left(D_{q}^{(p)} f\right)(z)}{\left(D_{q}^{(p-1)} f\right)(z)}}{(q-1) \frac{z\left(D_{q}^{(p)} f\right)(z)}{\left(D_{q}^{(p-1)} f\right)(z)}+2}-1\right] \\
& \leq(k+1)\left|\frac{(1+q) \frac{z\left(D_{q}^{(p)} f\right)(z)}{\left(D_{q}^{(p-1)} f\right)(z)}}{(q-1) \frac{z\left(D_{q}^{(p)} f\right)(z)}{\left(D_{q}^{(p-1)} f\right)(z)}+2}-1\right| \\
& =2(k+1)\left|\frac{z\left(D_{q}^{(p)} f\right)(z)-\left(D_{q}^{(p-1)} f\right)(z)}{(q-1) z\left(D_{q}^{(p)} f\right)(z)+2\left(D_{q}^{(p-1)} f\right)(z)}\right| \\
& =2(k+1)\left|\frac{[n+p]_{q} !}{\left.(q n+1]_{q}-1\right) a_{n+p} z^{n+1}}\right| \\
& \leq 2 \frac{\sum_{n=1}^{\infty} \frac{[n+1]_{q} !}{(q]_{q} !+\sum_{n=1}^{\infty} \frac{[n+p]_{q} !}{[n+1]_{q} !}\left\{(q-1)[n+1]_{q}+2\right\} a_{n+p} z^{n+1}} \mid}{(q+1)+\sum_{n=2}^{\infty} \frac{[n+p]_{q} !}{[n+1]_{q} !}\left|(q-1)[n+1]_{q}+2\right|\left|a_{n+p}\right|},
\end{aligned}
$$

the upper bound of the relation given by (3.3) is unity if

$$
\sum_{n=1}^{\infty} \frac{[n+p]_{q} !}{[n+1]_{q} !} \Lambda_{3} \cdot\left|a_{n+p}\right|<(1+q)
$$

where $\Lambda_{3}$ is given by (3.2). Consequently, proof is completed.

If in Theorem 3 , we put $p=1$ and $q \longrightarrow 1-$, then we get the following result.

Corollary 1 (See [11]) Any function $f \in \mathcal{A}$ of the form (1.1) belongs to the class $k-\mathcal{S}^{*} \mathcal{T}$ if it satisfies the inequality

$$
\sum_{n=1}^{\infty}\{(k+1) n+1\}\left|a_{n}\right|<1 .
$$

Theorem 2 A function $f \in \mathcal{A}(p)$ of the form (1.1) belongs to the function class $k-\mathcal{C}_{q}(p)$ if

$$
\sum_{n=2}^{\infty} \frac{[n+p]_{q} !}{[n]_{q} !} \Lambda_{3}\left|a_{n+p}\right|<(1+q)
$$

where $\Lambda_{3}$ is defined in (3.2). 
Proof We omit the details of the proof, since it easily follows by applying Theorem 1 in conjunction with Definition 11.

Theorem 3 A function $f \in \mathcal{A}(p)$ having series expansion (1.1) belongs to the class $k-\mathcal{K}_{q}^{*}(p)$ if

$$
\sum_{n=2}^{\infty}\left\{2(k+1) \Lambda_{1}+\Lambda_{2}\right\}<(1+q)
$$

where

$$
\Lambda_{1}=\left|b_{n+p}-[n+1]_{q} a_{n+p}\right|
$$

and

$$
\Lambda_{2}=\left|(1-q)[n+1]_{q} a_{n+p}-2 b_{n+p}\right| .
$$

Proof Assuming that (3.4) holds, it suffices to check that

$$
k\left|\frac{(1+q) \frac{z\left(D_{q}^{(p)} f\right)(z)}{\left(D_{q}^{(p-1)} g\right)(z)}}{(q-1) \frac{z\left(D_{q}^{(p)} f\right)(z)}{\left(D_{q}^{(p-1)} g\right)(z)}+2}-1\right|-\Re\left[\frac{(1+q) \frac{z\left(D_{q}^{(p)} f\right)(z)}{\left(D_{q}^{(p-1)} g\right)(z)}}{(q-1) \frac{z\left(D_{q}^{(p)} f\right)(z)}{\left(D_{q}^{(p-1)} g\right)(z)}+2}-1\right]<1 .
$$

We have

$$
\begin{aligned}
k \mid & \frac{(1+q) \frac{z\left(D_{q}^{(p)} f\right)(z)}{\left(D_{q}^{(p-1)} g\right)(z)}}{(q-1) \frac{z\left(D_{q}^{(p)} f\right)(z)}{\left(D_{q}^{(p-1)} g\right)(z)}+2}-1 \mid-\Re\left[\frac{(1+q) \frac{z\left(D_{q}^{(p)} f\right)(z)}{\left(D_{q}^{(p-1)} g\right)(z)}}{(q-1) \frac{z\left(D_{q}^{(p)} f\right)(z)}{\left(D_{q}^{(p-1)} g\right)(z)}+2}-1\right] \\
& \leq(k+1)\left|\frac{(1+q) \frac{z\left(D_{q}^{(p)} f\right)(z)}{\left(D_{q}^{(p-1)} g\right)(z)}}{(q-1) \frac{z\left(D_{q}^{(p)} f\right)(z)}{\left(D_{q}^{(p-1)} g\right)(z)}+2}-1\right| \\
& =2(k+1)\left|\frac{z\left(D_{q}^{(p)} f\right)(z)-2\left(D_{q}^{(p-1)} g\right)(z)}{(q-1) z\left(D_{q}^{(p)} f\right)(z)+2\left(D_{q}^{(p-1)} g\right)(z)}\right| \\
& =2(k+1)\left|\frac{\sum_{n=1}^{\infty} \frac{[n+p] q_{!} !}{[n+1]_{q} !}\left\{[n+1]_{q} a_{n+p}-b_{n+p}\right\} z^{n+1}}{(q+1)[p]_{q} ! z+\sum_{n=1}^{\infty} \frac{\left[n+p q_{q} !\right.}{[n+1]_{q} !}\left(\{-1)[n+1]_{q} a_{n+p}+2 b_{n+p}\right\} z^{n+1}}\right| \\
& \leq \frac{2(k+1) \sum_{n=1}^{\infty} \frac{[n+p] q}{[n+1]_{q} !}\left|[n+1]_{q} a_{n+p}-b_{n+p}\right|}{(q+1)[p]_{q} !-\sum_{n=2}^{\infty} \frac{[n+p] q}{[n+1]_{q} !}\left|(1-q)[n+1]_{q} a_{n+p}-2 b_{n+p}\right|} .
\end{aligned}
$$

The last expression in (3.7) is bounded above by 1 if

$$
\sum_{n=1}^{\infty}\left\{2(k+1) \Lambda_{1}+\Lambda_{2}\right\}<(1+q)
$$

where $\Lambda_{1}$ and $\Lambda_{2}$ are given by (3.5) and (3.6), respectively, which completes the proof. 
Theorem 4 A function from the class $\mathcal{A}(p)$ having series expansion (1.1) belongs to the class $k-\mathcal{C}_{q}^{*}(p)$ if

$$
\sum_{n=1}^{\infty}[n+1]_{q}\left\{2(k+1) \Lambda_{1}+\Lambda_{2}\right\}<(1+q)
$$

where $\Lambda_{1}$ and $\Lambda_{2}$ are respectively presented in (3.5) and (3.6).

Proof The proof of Theorem 4 follows easily by using Theorem 3 and Definition 13.

Theorem 5 Let $f \in k-\mathcal{S}_{q}^{*}(p)$ be of the form (1.1). Then

$$
\left|a_{n+p-1}\right| \leq \prod_{j=2}^{n} \frac{[j]_{q}\left\{2\left([j-1]_{q}-1\right)+(q+1) \delta_{k}\right\}}{2\left\{[j]_{q}-1\right\}[j+p-1]_{q}} \quad(n \in \mathbb{N} \backslash\{1\})
$$

Proof For $f \in k-\mathcal{S}_{q}^{*}(p)$, by definition we obtain

$$
\frac{z\left(D_{q}^{(p)} f\right)(z)}{\left(D_{q}^{(p-1)} f\right)(z)}=\psi(z)
$$

where

$$
\psi(z) \prec \frac{2 p_{k}(z)}{(1+q)+(1-q) p_{k}(z)} .
$$

If

$$
p_{k}(z)=1+\delta_{k} z+\cdots,
$$

then after some appropriate computations, condition (3.10) can be written as

$$
\psi(z) \prec 1+\frac{(1+q)}{2} \delta_{k} z+\cdots,
$$

and if

$$
\psi(z)=1+\sum_{n=1}^{\infty} \psi_{n} z^{n}
$$

then by application of Lemma 1 in conjunction with (3.11) and (3.12) we have

$$
\left|\psi_{n}\right| \leq \frac{(1+q)}{2}\left|\delta_{k}\right| \quad(n \in \mathbb{N}) .
$$

Now from (3.9) we set

$$
z\left(D_{q}^{(p)} f\right)(z)=\left(D_{q}^{(p-1)} f\right)(z) \psi(z)
$$


which implies that

$$
\begin{aligned}
{[p]_{q} ! z+\sum_{n=1}^{\infty} \frac{[n+p]_{q} !}{[n+1]_{q} !} a_{n+p} z^{n+1}=} & \left([p]_{q} ! z+\sum_{n=2}^{\infty} \frac{[n+p]_{q} !}{[n+1]_{q} !} a_{n+p} z^{n+1}\right) \\
& \cdot\left(1+\sum_{n=1}^{\infty} \psi_{n} z^{n}\right) .
\end{aligned}
$$

Now the comparison of the corresponding coefficients of $z^{n}$ gives

$$
\frac{[n+p]_{q} !}{[n+1]_{q} !}\left([n+1]_{q}-1\right) a_{n+p}=\sum_{l=1}^{n} \frac{[n+p-l]_{q} !}{[n+1-l]_{q} !} a_{n+p-l} c_{l}, \quad a_{p}=1 .
$$

Equivalently,

$$
\left|a_{n+p}\right| \leq \frac{[n+1]_{q} !}{[n+p]_{q} !\left([n+1]_{q}-1\right)} \sum_{l=1}^{n} \frac{[n+p-l]_{q} !}{[n+1-l]_{q} !}\left|a_{n+p-l}\right|\left|c_{l}\right|, \quad a_{p}=1 .
$$

Moreover, by (3.13) we have

$$
\left|a_{n+p}\right| \leq \frac{\delta_{k}(q+1)[n+1]_{q} !}{2[n+p]_{q} !\left([n+1]_{q}-1\right)} \sum_{l=1}^{n-1} \frac{[p+l]_{q} !}{[l+1]_{q} !}\left|a_{p+l}\right|, \quad a_{p}=1 .
$$

Now, using Lemma 2, we have

$$
\left|a_{n+p-1}\right| \leq \prod_{j=2}^{n} \frac{[j]_{q}\left\{2\left([j]_{q}-1\right)+\delta_{k}(q+1)\right\}}{2\left\{[j]_{q}-1\right\}[j+p-1]_{q}}
$$

Specifically, for instance, setting $p=1$ and letting $q \longrightarrow 1-$, we obtain the estimate on the $n$th coefficient of the class $k-\mathcal{S}^{*} \mathcal{T}$, settled by Wisniowska and Kanas as follows.

Corollary 2 (See [11]) For an analytic function $f \in k-\mathcal{S}^{*} \mathcal{T}$, we have

$$
\left|a_{n}\right| \leq \prod_{j=0}^{n-2} \frac{j(j-2)+\delta_{k}}{(j-1)(j)} \quad(n \in \mathbb{N} \backslash\{1\}) .
$$

Theorem 6 Let $f \in k-\mathcal{C}_{q}(p)$ be of the form (1.1). Then

$$
\left|a_{n+p-1}\right| \leq \frac{1}{[n+p]_{q}} \prod_{j=2}^{n} \frac{[j]_{q}\left\{2\left([j-1]_{q}-1\right)+(q+1) \delta_{k}\right\}}{2\left\{[j]_{q}-1\right\}[j+p-1]_{q}} \quad(n \in \mathbb{N} \backslash\{1\}) .
$$

Proof The proof of Theorem 6 follows easily by using Definition 11 and Theorem 5 . 
Theorem 7 Let $f \in k-\mathcal{K}_{q}^{*}$ be of the form (1.1). Then

$$
\begin{aligned}
\left|a_{n+p-1}\right| \leq & \frac{[n]_{q} !}{[n+p]_{q} !}\left(\prod_{j=0}^{n-2} \frac{[j]_{q}\left\{2\left([j-1]_{q}-1\right)+\delta_{k}(q+1)\right\}}{2\left\{[j]_{q}-1\right\}[j+p-1]_{q}}\right) \\
& +\frac{(q+1)\left|\delta_{k}\right|}{2[n]_{q}} \sum_{j=1}^{n-1} \prod_{j=0}^{n-2} \frac{\left|\delta_{k}(q+1)+2 q[j]_{q}\right|}{2 q[j+1]_{q}} \quad(n \in \mathbb{N} \backslash\{1\}) .
\end{aligned}
$$

Proof By definition, for a function $f$ belonging to $k-\mathcal{K}_{q}(p)$, we have that

$$
\frac{z\left(D_{q}^{(p)} f\right)(z)}{\left(D_{q}^{(p-1)} g\right)(z)}=\psi(z),
$$

where

$$
g(z)=z^{p}+\sum_{n=1}^{\infty} b_{n+p} z^{n+p}
$$

and that

$$
\psi(z) \prec \frac{2 p_{k}(z)}{(1+q)+(1-q) p_{k}(z)} .
$$

If

$$
p_{k}(z)=1+\delta_{k} z+\cdots,
$$

then after some convenient computations, condition (3.18) can be written as

$$
\psi(z) \prec 1+\frac{(1+q)}{2} \delta_{k} z+\cdots,
$$

and if

$$
\psi(z)=1+\sum_{n=1}^{\infty} \psi_{n} z^{n},
$$

then applying Lemma 1 in conjunction with (3.19) and (3.20), we get

$$
\left|\psi_{n}\right| \leq \frac{(1+q)}{2}\left|\delta_{k}\right| \quad(n \in \mathbb{N})
$$

Next, equation (3.17) may be written as

$$
z\left(D_{q}^{(p)} f\right)(z)=\left(D_{q}^{(p-1)} g\right)(z) \psi(z)
$$


and using the series form, we get

$$
\begin{aligned}
{[p]_{q} ! z+\sum_{n=1}^{\infty} \frac{[n+p]_{q} !}{[n+1]_{q} !} a_{n+p} z^{n+1}=} & \left([p]_{q} ! z+\sum_{n=2}^{\infty} \frac{[n+p]_{q} !}{[n+1]_{q} !} b_{n+p} z^{n+1}\right) \\
& \cdot\left(1+\sum_{n=1}^{\infty} \psi_{n} z^{n}\right) .
\end{aligned}
$$

Next, the comparison of the corresponding coefficients of $z^{n}$ yields

$$
\frac{[n+p]_{q} !}{[n+1]_{q} !}\left([n+1]_{q} a_{n+p}-b_{n+p}\right)=\sum_{l=1}^{n} \frac{[n+p-l]_{q} !}{[n+1-l]_{q} !} a_{n+p-l} c_{l} \quad\left(a_{p}=1\right) .
$$

This implies that

$$
\frac{[n+p]_{q} !}{[n+1]_{q} !}[n+1]_{q}\left|a_{n+p}\right| \leq\left|b_{n+p}\right|+\sum_{j=1}^{n-1}\left|a_{n+p-l}\right|\left|c_{l}\right| \quad\left(a_{1}=1\right) .
$$

Moreover, using Theorem 5 and (3.21), we get

$$
\begin{aligned}
\left|a_{n+p}\right| \leq & \frac{[n]_{q} !}{[n+p]_{q} !}\left(\prod_{j=0}^{n-2} \frac{[j]_{q}\left\{2\left([j-1]_{q}-1\right)+\delta_{k}(q+1)\right\}}{2\left\{[j]_{q}-1\right\}[j+p-1]_{q}}\right) \\
& +\frac{[n+1]_{q} !(q+1)\left|\delta_{k}\right|}{2[n+1]_{q}[n+p]_{q} !} \sum_{j=1}^{n-1} \prod_{j=0}^{n-2} \frac{[j]_{q}\left\{2\left([j-1]_{q}-1\right)+\delta_{k}(q+1)\right\}}{2\left\{[j]_{q}-1\right\}[j+p-1]_{q}} .
\end{aligned}
$$

Thus we have proved the statement of Theorem 7 .

Putting $p=1$ and letting $q \longrightarrow 1$ - in Theorem 7, we obtain the estimates of the $n$th coefficients of the functions from the class $k-\mathcal{U} \mathcal{K}$, given by Noor et al.

Corollary 3 (See [18]) Let $f \in k-\mathcal{U K}$ be of the form (1.1). Then

$$
\left|a_{n}\right| \leq \frac{\left(\left|\delta_{k}\right|\right)_{n-1}}{n !}+\frac{\left|\delta_{k}\right|}{n} \sum_{j=1}^{n-1} \frac{\left(\left|\delta_{k}\right|\right)_{j-1}}{(j-1) !} \quad(n \in \mathbb{N} \backslash\{1\}) .
$$

Further, setting

$$
k=0=p-1
$$

in Theorem 7, then $\delta_{k}=2$, and letting $q \longrightarrow 1-$, we get the known result by Kaplan et al.

Corollary 4 ([12]) Let $f \in \mathcal{K}$ be an analytic function. Then

$$
\left|a_{n}\right| \leq n \quad(n \in \mathbb{N} \backslash\{1\}) .
$$


Theorem 8 Let $f \in k-\mathcal{C}_{q}^{*}(p)$ with series expansion (1.1). Then

$$
\begin{aligned}
\left|a_{n}\right| \leq & \frac{[n]_{q}^{2} !}{[n+p]_{q}^{2 !}}\left(\prod_{j=0}^{n-2} \frac{[j]_{q}\left\{2\left([j-1]_{q}-1\right)+\delta_{k}(q+1)\right\}}{2\left\{[j]_{q}-1\right\}[j+p-1]_{q}}\right)+\frac{(q+1)\left|\delta_{k}\right|}{2[n+p]_{q} !} \\
& \cdot \sum_{j=1}^{n-1} \prod_{j=0}^{n-2} \frac{[j]_{q}\left\{2\left([j-1]_{q}-1\right)+\delta_{k}(q+1)\right\}}{2\left\{[j]_{q}-1\right\}[j+p-1]_{q}} \quad(n \in \mathbb{N} \backslash\{1\}) .
\end{aligned}
$$

Proof Using Theorem 7 and Definition 13 immediately yields the proof.

\author{
Acknowledgements \\ The work here is supported by UKM Grant: FRGS/1/2019/STG06/UKM/01/1.
}

Funding

UKM Grant: FRGS/1/2019/STG06/UKM/01/1.

\title{
Availability of data and materials
}

Not applicable.

\section{Competing interests}

The authors declare that they have no competing interests.

\section{Authors' contributions}

All authors jointly worked on the results, and they read and approved the final manuscript.

\section{Author details}

'Department of Mathematics, Abbottabad University of Science and Technology, Abbottabad 22010, Pakistan. ${ }^{2}$ Government Akhtar Nawaz Khan (Shaheed) Degree College, KTS, Haripur 22620, Pakistan. ${ }^{3}$ Department of Mathematics and Statistics, University of Victoria, Victoria, British Columbia V8W 3R4, Canada. ${ }^{4}$ Department of Medical Research, China Medical University Hospital, China Medical University, Taichung 40402, Taiwan, Republic of China. ${ }^{5}$ Department of Mathematics and Informatics, Azerbaijan University, 71 Jeyhun Hajibeyli Street, AZ1007 Baku, Azerbaijan. ${ }^{6}$ Department of Mathematical Sciences, Faculty of Sciences and Technology, Universiti Kebangsaan Malaysia, 43600 Bangi, Selangor, Malaysia.

\section{Publisher's Note}

Springer Nature remains neutral with regard to jurisdictional claims in published maps and institutional affiliations.

Received: 12 March 2020 Accepted: 18 June 2020 Published online: 01 July 2020

\section{References}

1. Acu, M.: On a subclass of n-uniformly close-to-convex functions. Gen. Math. 14, 55-64 (2006)

2. Baricz, Á., Swaminathan, A.: Mapping properties of basic hypergeometric functions. J. Class. Anal. 5, 115-128 (2014)

3. Duren, P.L.: Univalent Functions. Grundlehren der Mathematischen Wissenschaften, vol. 259. Springer, New York (1983)

4. Goodman, A.W.: On uniformly convex functions. Ann. Pol. Math. 56, 87-92 (1991)

5. Goodman, A.W.: On uniformly starlike functions. J. Math. Anal. Appl. 155, 364-370 (1991)

6. Ismail, M.E.-H., Merkes, E., Styer, D.: A generalization of starlike functions. Complex Var. Theory Appl. 14, 77-84 (1990)

7. Jackson, F.H.: On q-definite integrals. Q. J. Pure Appl. Math. 41, 193-203 (1910)

8. Jackson, F.H.: q-Difference equations. Am. J. Math. 32, 305-314 (1910)

9. Kanas, S.: Coefficient estimates in subclasses of the Carathéodory class related to conical domains. Acta Math. Univ. Comen. 74, 149-161 (2005)

10. Kanas, S., Wiśniowska, A.: Conic regions and k-uniform convexity. J. Comput. Appl. Math. 105, 327-336 (1999)

11. Kanas, S., Wiśniowska, A.: Conic domains and starlike functions. Rev. Roum. Math. Pures Appl. 45, 647-657 (2000)

12. Kaplan, W.: Close-to-convex Schlicht functions. Mich. Math. J. 1, 169-185 (1952)

13. Khan, Q., Arif, M., Raza, M., Srivastava, G., Tang, H.: Some applications of a new integral operator in $q$-analog for multivalent functions. Mathematics 7(12), Article ID 1178 (2019)

14. Ma, W., Minda, W.A.: A unified treatment of some special classes of univalent functions. In: Proceedings of the Conference on Complex Analysis. Conf. Proc. Lecture Notes Anal., pp. 157-169. Int. Press, Cambridge (2019)

15. Mahmood, S., Ahmad, Q.Z., Srivastava, H.M., Khan, N., Khan, B., Tahir, M.: A certain subclass of meromorphically q-starlike functions associated with the Janowski functions. J. Inequal. Appl. 2019, Art. 88 (2019)

16. Mahmood, S., Raza, N., AbuJarad, E.S., Srivastava, G., Srivastava, H.M., Malik, S.N.: Geometric properties of certain classes of analytic functions associated with a q-integral operator. Symmetry 11(5), Article ID 719 (2019)

17. Mahmood, S., Srivastava, H.M., Khan, N., Ahmad, Q.Z., Khan, B., Ali, I.: Upper bound of the third Hankel determinant for a subclass of q-starlike functions. Symmetry 11, Article ID 347 (2019) 
18. Noor, K.I., Arif, M., Ul-Haq, W.: On k-uniformly close-to-convex functions of complex order. Appl. Math. Comput. 215 , 629-635 (2009)

19. Noor, K.I., Thomas, D.K.: Quasi convex univalent functions. Int. J. Math. Math. Sci. 3, 255-266 (1980)

20. Raghavendar, K., Swaminathan, A.: Close-to-convexity of basic hypergeometric functions using their Taylor coefficients. J. Math. Appl. 35, 111-125 (2012)

21. Rehman, M.S., Ahmad, Q.Z., Srivastava, H.M., Khan, B., Khan, N.: Partial sums of generalized q-Mittag-Leffler functions. AIMS Math. 5(1), 408-420 (2019)

22. Rogosinski, W.: On the coefficients of subordinate functions. Proc. Lond. Math. Soc. 48, 48-82 (1943)

23. Rønning, F.: On starlike functions associated with parabolic regions. Ann. Univ. Mariae Curie-Skłodowska, Sect. A 45 , 117-122 (1991)

24. Rønning, F.: Uniformly convex functions and a corresponding class of starlike functions. Proc. Am. Math. Soc. 118, 189-196 (1993)

25. Sahoo, S.K., Sharma, N.L.: On a generalization of close-to-convex functions. Ann. Pol. Math. 113, 93-108 (2015)

26. Srivastava, H.M.: Univalent functions, fractional calculus, and associated generalized hypergeometric functions. In: Srivastava, H.M., Owa, S. (eds.) Univalent Functions, Fractional Calculus, and Their Applications, pp. 329-354. Horwood, Chichester (1989)

27. Srivastava, H.M.: Operators of basic (or $q$-) calculus and fractional $q$-calculus and their applications in geometric function theory of complex analysis. Iran. J. Sci. Technol. Trans. A, Sci. 44, 327-344 (2020)

28. Srivastava, H.M., Ahmad, Q.Z., Khan, N., Khan, N., Khan, B.: Hankel and Toeplitz determinants for a subclass of $q$-starlike functions associated with a general conic domain. Mathematics 7(2), 181 (2019)

29. Srivastava, H.M., Aouf, M.K., Mostafa, A.O.: Some properties of analytic functions associated with fractional $q$-calculus operators. Miskolc Math. Notes 20, 1245-1260 (2019)

30. Srivastava, H.M., Bansal, D.: Close-to-convexity of a certain family of $q$-Mittag-Leffler functions. J. Nonlinear Var. Anal. 1(1), 61-69 (2017)

31. Srivastava, H.M., Khan, B., Khan, N., Ahmad, Q.Z.: Coefficient inequalities for q-starlike functions associated with the Janowski functions. Hokkaido Math. J. 48, 407-425 (2019)

32. Srivastava, H.M., Khan, B., Khan, N., Ahmad, Q.Z., Tahir, M.: A generalized conic domain and its applications to certain subclasses of analytic functions. Rocky Mt. J. Math. 49(7), 2325-2346 (2019)

33. Srivastava, H.M., Raza, N., AbuJarad, E.S.A., Srivastava, G., AbuJarad, M.H.: Fekete-Szegö inequality for classes of $(p, q)$-starlike and $(p, q)$-convex functions. Rev. R. Acad. Cienc. Exactas Fís. Nat., Ser. A Mat. 113, 3563-3584 (2019)

34. Srivastava, H.M., Tahir, M., Khan, B., Ahmad, Q.Z., Khan, N.: Some general families of $q$-starlike functions associated with the Janowski functions. Filomat 33(9), 2613-2626 (2019)

35. Srivastava, H.M., Tahir, M., Khan, B., Ahmad, Q.Z., Khan, N.: Some general classes of $q$-starlike functions associated with the Janowski functions. Symmetry 11, 292 (2019)

36. Uçar, H.E.Ö.: Coefficient inequality for q-starlike functions. Appl. Math. Comput. 276, 122-126 (2016)

\section{Submit your manuscript to a SpringerOpen ${ }^{\circ}$ journal and benefit from:}

- Convenient online submission

- Rigorous peer review

- Open access: articles freely available online

- High visibility within the field

- Retaining the copyright to your article

Submit your next manuscript at $\boldsymbol{\nabla}$ springeropen.com 